\title{
Prevention Of Inflammatory Damage In Hypothalamus by Supplementation With w3 Fatty Acid in a Sepsis Model: The Role of the Cholinergic Receptor
}

Ísis C. A. Martins ${ }^{\star}$, Letícia S. Contieri, Camilla M. de Souza, Camila L. do Amaral, Alana C.C. Veras, Anelise C. P. Souza, Marcio A. Torsoni.

\begin{abstract}
The cholinergic anti-inflammatory pathway has been studied in relation to its immunomodulatory function and effects on infectious processes, including sepsis. Studies from our laboratory suggest the damage of the cholinergic antiinflammatory response in animals fed with short term HFD. Omega3 polyunsaturated fatty acids have an important antiinflammatory role through the interaction with GPR-120 type receptors. To evaluate if the supplementation with w3 fatty acid prevents inflammatory damage in the hypothalamus in a model of sepsis, swiss male mice were randomly assigned to be fed with normal chow diet (SC) or high fat diet (HFD) for 3 days or orally supplemented during 17 days with w3 fatty acid and fed with HFD for the last 3 days (HFDw3). Sepsis was induced by surgery of cecal ligation and puncture (CLP). At baseline, w3 supplementation was not able to reverse the decrease of the protein content of the $\alpha 7$ nicotinic acetylcholine receptor $(\alpha 7 n A C h R)$ caused by HFD in the hypothalamus. After CLP surgery, mice that received w3 fatty acid supplementation prior to short term HFD showed increased protein content of a7nAChR in the hypothalamus and increased survival rate compared to mice fed only with HFD. These results show that the supplementation with w3 fatty acid may be able to prevent damage in a model of sepsis.
\end{abstract}

\section{Key words: \\ a7nAChR, hypothalamus, Omega 3.}

\section{Introduction}

The cholinergic anti-inflammatory pathway has been studied in relation to its immunomodulatory function and effects on infectious processes, including sepsis ${ }^{1}$. Studies from our laboratory suggest the damage of the cholinergic anti-inflammatory response in animals fed with short term HFD². Omega3 polyunsaturated fatty acids have an important anti-inflammatory role through the interaction with GPR-120 type receptors ${ }^{3}$.

\section{Results and Discussion}

Swiss male mice were randomly assigned to be fed with normal chow diet (SC) or high fat diet (HFD) for 3 days or orally supplemented during 17 days with w3 fatty acid and fed with HFD for the last 3 days (HFDw3). Sepsis was induced by surgery of cecal ligation and puncture (CLP). At baseline, w3 supplementation was not able to reverse the decrease of the protein content of receptor $\alpha 7 n A C h R$ caused by HFD. We also did not observe a significant difference in the amount of mRNA of cytokines and chemokines, except for IL-6, which was significantly lower in HFD-fed mice compared to SC, and HFDw3 group was able to reverse this. This may be explained by the low rate of inflammation characteristic of the short time exposure of the diet, which would not be able to significantly modulate inflammatory markers. ${ }^{4}$

To identify whether HFD-fed mice are more likely to develop sepsis, the survival curves of SC and HFD mice were compared after the CLP surgery. Mice that consumed short term HFD + previous supplementation with w3 fatty acid showed an increased survival rate compared to short term HFD, whereas HFD died faster compared to SC mice. At the same time, we tested a doubled dose of supplementation, and there was a tendency to increase the protein content of $\alpha 7 n A C h R$. Therefore, we followed the analysis with the new dose.

After CLP surgery, w3 supplementation increased the protein content of $\alpha 7 \mathrm{nAChR}$ compared to SHAM and SCCLP group, but not compared to HFD-CLP group. These results suggest that previous supplementation with w3 fatty acid increases the content of $\alpha 7 n A C h R$, but does not reverse the damage caused by HFD.

Image 1. Survival rate

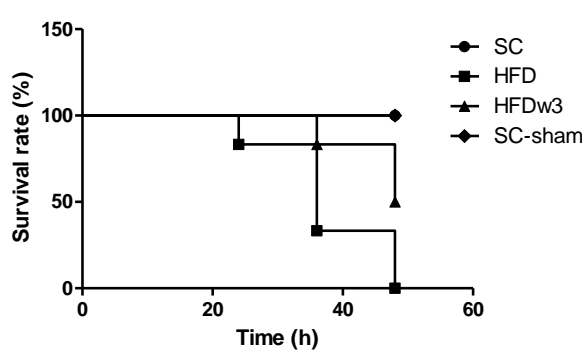

Conclusions

At baseline, the previous supplementation with w3 fatty acid was not able to prevent the damage caused by HFD in the hypothalamus.

After the CLP surgery, the previous supplementation with w3 fatty acid was able to increase the content of receptor $\alpha 7 n A C h R$ and reduce the risk of sepsisassociated mortality.

\section{Acknowledgement}

CNPq, FAPESP 2016/23484-1.

${ }^{1}$ WANG, XianFeng et al. Activation of the cholinergic antiinflammatory pathway ameliorates obesity-induced inflammation and insulin resistance. Endocrinology, v. 152, n. 3, p. 836-846, 2011.

${ }^{2}$ SOUZA, A.C.P. et al. Short-term high-fat diet consumption reduces hypothalamic expression of the nicotinic acetylcholine receptor $\alpha 7$ subunit $(\alpha 7 \mathrm{nAChR})$ and affects the anti-inflammatory response in a mouse model of sepsis. Frontiers in Immunology, v. 10, p. 565, 2019.

${ }^{3} \mathrm{OH}$, Da Young et al. GPR120 is an omega-3 fatty acid receptor mediating potent anti-inflammatory and insulin-sensitizing effects. Cell, v. 142, n. 5, p 687-698, 2010

${ }^{4}$ Ding, Shengli et al. High-fat diet: bacteria interactions promote intestinal inflammation which precedes and correlates with obesity and insulin resistance in mouse. PloS one, v. 5, n. 8, p. e12191, 2010. 